\title{
Web Impact Factor for Malaysian Public Universities
}

\author{
Abdul Arif and N. A. Ismail
}

\begin{abstract}
This paper evaluates Public Universities in Malaysia based on the webometric perspective. Web Impact Factor (WIF) evaluation was carried out by using Majestic SEO and Google Search engine. It is found that UMS have the highest Average WIF with 13.013 followed by UMK with 2.670 WIF and in the third place is UPNM with 1.289 WIF.
\end{abstract}

Index Terms-Malaysian public universities, web impact factor, webometrics.

\section{INTRODUCTION}

There are two types of university in Malaysia, namely public university and private university. All public universities have their personal websites that are normally used to spread intended information to the general community. However, not much research has been done on the quality of the websites. Since these websites are the primary information sources in this technological era, related research is important to identify its influence and quality. Thus, this research has been conducted to evaluate the Web Impact Factor (WIF) of the websites of public universities in Malaysia.

All of the public universities in have their own websites and on average, most of the websites have more than 10,000 pages. To manually evaluate the quality and performance of these websites thus become a tedious task and to overcome this problem, Peter Ingwersen proposed the Web Impact Factor (WIF) [1]. WIF adapts the principal concept of the Journal Impact Factor which is widely used to evaluate the performance of journals. WIF for a website, on the other hand, intends to evaluate the performance of the website using In-link Count and Page Count. The formula for calculating WIF was further improved in 2001 where Peter Ingwersen changed the In-link Count to External In-link Count [2].

\section{LITERATURE REVIEW}

Many researchers had done studies in the webometrics field [3]-[7]. For example, Vaughan did a study on business websites [7]. Noruzi (2006) also performed a study using the

Manuscript received July 29, 2012; revised November 18, 2012. This work was supported in part by the Universiti Teknologi Malaysia under Research Grant VOT 01J14.

Abdul Arif is a researcher in Faculty of Computer Science \& Information System. Graduated in the Mecahnical Engineering field, he is now pursuing Master of Computer Science in Universiti Teknologi Malaysia, Johor, Malaysia (e-mail: misterpah@gmail.com).

N. A. Ismail is currently the Deputy Director of Corporate Affairs (Web Director) and a senior lecturer in Universiti Teknologi Malaysia (e-mail: azman@utm.my).
WIF [5]. Farzaneh Aminpour did a webometrics analysis on medical science universities in year 2009 [8]. In addition, Samir Kumar Jalal carried out a study also using WIF on some Indian universities [9]. Recently, in 2011, Handaru Jati had done similar study on Indonesian universities [10]. At the same time, Md. Anwarul Islam did the same on Bangladeshi universities [11].

Such research may seem easy, but it is crucial for the general public and concerned parties like the Ministry of Higher Education (MOHE) to evaluate the standard of a university's website. By dividing the In-link Count with the Page Count, the WIF can be obtained. The formula adjustment carried out in year 2001 had further enhanced the accuracy of the WIF obtained thereafter.

Yahoo Site Explorer had been the major tool to calculate the External In-link Count for most webometrics research [4]. However, in year 2011, Yahoo had decided to terminate the related services due to the merging of Yahoo and Microsoft [12]. There are various reactions towards this decision; most of the users are not happy as the Yahoo Site Explorer has been the most preferred service for calculating External In-link Count [13]. In fact, it has been used even by business companies to evaluate the selling points, popularity and credibility of competitors. The termination of such important service means that the users will need to find other resources and this has helped the Majestic SEO to surge into popularity.

\section{METHOD}

This study collected the required data using the Google Search Engine and the Majestic SEO. The data were collected within the same month - July 2012. To obtain the Page Count for a certain website, the two search engines were utilized simultaneously. In Google, the keyword "site:domain. Top Level Domain" was used to get the Page Count while in Majestic SEO; the Page Count was taken from the "Indexed URL" parameter. Since the Majestic SEO has two options for data collection, the "Fresh Index" was chosen for this study to ensure that all data collected were the latest data at that time.

To obtain the External In-link Count, Google could not be used because it does not provide any related service. Therefore, Majestic SEO became the major service. In this case, the "External Backlink" parameter from the "Fresh Index" data was taken as the External In-link Count. After the collection of these two data (Page Count and External In-link Count), the WIF was calculated. This can be done using the formula in (1).

$$
W I F=\frac{\text { External In }- \text { link count }}{\text { Page count }}
$$

As this study was focused on the public universities in 